\title{
On unification of gravity and gauge interactions
}

\author{
Ali H. Chamseddine ${ }^{a, c}$ and Viatcheslav Mukhanov ${ }^{b}$ \\ ${ }^{a}$ Physics Department, American University of Beirut, \\ Beirut, Lebanon \\ ${ }^{b}$ Theoretical Physics, Ludwig Maxmillians University, \\ Theresienstr. 37, 80333 Munich, Germany \\ ${ }^{c}$ Institut des Hautes Études Scientifiques (I.H.E.S.), \\ F-91440 Bures-sur-Yvette, France \\ E-mail: chams@aub.edu.1b, viatcheslav.Mukhanov@lmu.de
}

ABSTRACT: Considering a higher dimensional Lorentz group as the symmetry of the tangent space, we unify gravity and gauge interactions in a natural way. The spin connection of the gauged Lorentz group is then responsible for both gravity and gauge fields, and the action for the gauged fields becomes part of the spin curvature squared. The realistic group which unifies all known particles and interactions is the $\mathrm{SO}(1,13)$ Lorentz group whose gauge part leads to $\mathrm{SO}(10)$ grand unified theory and contains double the number of required fermions in the fundamental spinor representation. We briefly discuss the Brout-Englert-Higgs mechanism which breaks the $\mathrm{SO}(1,13)$ symmetry first to $\mathrm{SO}(1,3) \times \mathrm{SU}(3) \times \mathrm{SU}(2) \times \mathrm{U}(1)$ and further to $\mathrm{SO}(1,3) \times \mathrm{SU}(3) \times \mathrm{U}(1)$ and gives very heavy masses to half of the fermions leaving the others with light masses.

Keywords: Gauge Symmetry, Space-Time Symmetries, Classical Theories of Gravity, GUT

ArXiv EPrint: 1602.02295 


\section{Contents}

1 Introduction 1

2 Tangent group 1

3 Curvature 5

$\begin{array}{llr}4 & \text { Fermions } & 9\end{array}$

5 Conclusions 11

\section{Introduction}

In General Relativity the Lorentz group is realized as a local symmetry of the tangent manifold. There exists no spinor representations of the diffeomorphisms and this dictates the use of this local symmetry in curved space-time. Usually the dimension of the tangent space is taken to be equal to the dimension of the curved manifold and the Lorentz symmetry is then simply a manifestation of the equivalence principle for spaces without torsion. Considering the group of local Lorentz transformations in tangent space, we can reformulate General Relativity as a gauge theory where the gauge fields are the spinconnections. If the dimensions of space time and tangent space are the same, the gauge fields (spin-connections) simply encode the same amount of information about dynamics of the gravitational field as the affine connections and nothing more. However, the dimension of the tangent group must not necessarily be the same as the dimension of the manifold [1]. In [2] we have shown that the metricity condition have unambiguous solution also in the case when the tangent space of $4 \mathrm{~d}$ manifold is five dimensional and corresponds to the de Sitter group (5d Lorentz group). In such case the theory is also completely equivalent to General Relativity. In this paper we consider the tangent space with more than five dimensions and show that this allows us to unify gauge theories with gravity in terms of higher dimensional gauged Lorentz groups. The gauge transformations are then realized as subgroup of the tangent Lorentz group and the spinors describing matter are "unified" all being in the fundamental representation of this higher dimensional Lorentz group. The realistic group which unifies all particles within one family is $\mathrm{SO}(1,13)$ and naturally leads to Einstein gravity with the SO (10) gauge group being, however, not entirely equivalent to the $\mathrm{SO}(10)$ grand unified theory.

\section{Tangent group}

Let us consider a 4-dimensional manifold and assume that at every point of this manifold there is real $N$-dimensional tangent space spanned by linearly independent vectors $\mathbf{v}_{A}$, 
where $A=1,2 \ldots N$. Assuming that $N \geq 4$, the coordinate basis vectors $\mathbf{e}_{\alpha} \equiv \partial / \partial x^{\alpha}$, where $\alpha=1, \ldots 4$, span 4 -dimensional (sub)space in this space. Next we define the scalar product in the tangent space and take vectors $\mathbf{v}_{A}$ to be orthonormal with respect to the "Minkowski matrix" $\eta_{A B}(-,+, \ldots,+)$

$$
\mathbf{v}_{A} \cdot \mathbf{v}_{B}=\eta_{A B}
$$

The Lorentz transformations

$$
\tilde{\mathbf{v}}_{A}=\Lambda_{A}{ }^{B} \mathbf{v}_{B}, \quad \Lambda_{A}{ }^{C} \eta_{C D} \Lambda_{A}{ }^{D}=\eta_{A B}
$$

preserve the orthogonality of the basis vectors $\mathbf{v}_{A}, \tilde{\mathbf{v}}_{A} \cdot \tilde{\mathbf{v}}_{B}=\eta_{A B}$. The scalar product of coordinate basis vectors, which also reside in the tangent space, induces the metric in the 4-dimensional manifold

$$
\mathbf{e}_{\alpha} \cdot \mathbf{e}_{\beta}=g_{\alpha \beta}\left(x^{\gamma}\right)
$$

Expanding $\mathbf{e}_{\alpha}$ in $\mathbf{v}_{A}$-basis we have

$$
\mathbf{e}_{\alpha}=e_{\alpha}^{A} \mathbf{v}_{A}
$$

where the coefficients of the expansion $e_{\alpha}^{A}$ are the vielbiens (or soldering forms). Substituting in (2.3) we obtain the following expression for the metric $g_{\alpha \beta}$

$$
g_{\alpha \beta}=e_{\alpha}^{A} e_{\beta}^{B} \eta_{A B}=e_{\alpha}^{A} e_{A \beta} .
$$

Hereafter, we always raise and lower tangent space indices with Minkowski metric $\eta_{A B}$. Next we consider parallel transport on the manifold relating vectors in the "nearby" tangent spaces. The affine and spin-connections determining the rules of parallel transport for coordinate basis vectors and vielbiens are defined by

$$
\nabla_{\mathbf{e}_{\beta}} \mathbf{e}_{\alpha} \equiv \nabla_{\beta} \mathbf{e}_{\alpha}=\Gamma_{\alpha \beta}^{\nu} \mathbf{e}_{\nu}, \quad \nabla_{\beta} \mathbf{v}_{A}=-\omega_{\beta A}{ }^{B} \mathbf{v}_{B},
$$

where $\nabla_{\beta}$ is the derivative along a coordinate basis vector $\mathbf{e}_{\beta}$. For example when $\nabla_{\beta}$ is applied to a scalar function $f$ it gives $\nabla_{\beta} f=\partial f / \partial x^{\beta}$. Notice that $\eta_{A B}$ and $g_{\alpha \beta}$ as defined in (2.1) and (2.3) must be considered as the sets of scalar functions and, hence, $\nabla_{\beta} \eta_{A B}=0$, $\nabla_{\gamma} g_{\alpha \beta}=\partial g_{\alpha \beta} / \partial x^{\gamma} \equiv \partial_{\gamma} g_{\alpha \beta}{ }^{1}$.

Given $\eta_{A B}, g_{\alpha \beta}$ and $e_{\alpha}^{A}$ let us derive the consistency (metricity) conditions for the connections. Taking derivative of equation (2.1) and using (2.6) we obtain

$$
\left(\nabla_{\alpha} \mathbf{v}_{A}\right) \cdot \mathbf{v}_{B}+\mathbf{v}_{A} \cdot\left(\nabla_{\alpha} \mathbf{v}_{B}\right)=-\omega_{\beta A B}-\omega_{\beta B A}=\nabla_{\alpha} \eta_{A B}=0
$$

i.e., the spin-connection should be antisymmetric in tangent indices, $\omega_{\beta A B}=-\omega_{\beta B A}$. Applying $\nabla_{\beta}$ to

$$
e_{A \alpha}=\left(\mathbf{v}_{A} \cdot \mathbf{e}_{\alpha}\right)
$$

one gets

$$
\partial_{\beta} e_{A \alpha}=\left(\nabla_{\beta} \mathbf{v}_{A}\right) \cdot \mathbf{e}_{\alpha}+\mathbf{v}_{A} \cdot\left(\nabla_{\alpha} \mathbf{e}_{\alpha}\right)
$$

\footnotetext{
${ }^{1}$ We use the notations and methods of Misner, Thorne, Wheeler [4], in particular, Chapters 9 and 10.
} 
or using definitions in (2.6)

$$
\partial_{\beta} e_{A \alpha}=-\omega_{\beta A}^{B} e_{B \alpha}+\Gamma_{\alpha \beta}^{\nu} e_{A \nu} .
$$

Hereafter we assume that the space-time is torsion-free, that is, $\Gamma_{\alpha \beta}^{\nu}=\Gamma_{\beta \alpha}^{\nu}$. In this case, $16 N$ equations (2.10) can be solved to express 40 affine connections $\Gamma_{\alpha \beta}^{\nu}$ and $2 N(N-1)$ spin-connections $\omega_{\beta A B}$ in terms of the derivatives of the soldering forms $\partial_{\beta} e_{A \alpha}$. The number of equations matches the number of connections to be determined only if the dimension of the tangent space is equal either to $N=4$ or $N=5$ [2]. For $N \geqslant 6$ the number of equation in (2.10) is less than the number of unknown connections and $2 N^{2}-18 N+40=2(N-4)(N-5)$ variables remain undetermined by soldering forms. Let $N=n+4$, then the number of unconstrained components of the spin-connections $\omega_{\beta A} B$ is $2 n(n-1)$ which matches the number of $\mathrm{SO}(n)$ gauge fields. As we will see this allows us to account for the gauge transformations which become unified with gravity for higher dimensional gauged Lorentz group of the tangent space. Considering

$$
\partial_{\gamma} g_{\alpha \beta}=\partial_{\gamma}\left(e_{\alpha}^{A} e_{A \beta}\right)=\left(\partial_{\gamma} e_{\alpha}^{A}\right) e_{A \beta}+e_{\alpha}^{A}\left(\partial_{\gamma} e_{A \beta}\right)
$$

and substituting in the right hand side the expression for $\partial_{\gamma} e_{\alpha}^{A}$ from (2.10) we find

$$
\Gamma_{\alpha \gamma}^{\nu} g_{\nu \beta}+\Gamma_{\beta \gamma}^{\nu} g_{\alpha \nu}=\partial_{\gamma} g_{\alpha \beta}
$$

In the absence of torsion, $\Gamma_{\alpha \beta}^{\nu}=\Gamma_{\beta \alpha}^{\nu}$, these equations are solved unambiguously, to give the well known Christoffel connection

$$
\Gamma_{\alpha \beta}^{\gamma}=\frac{1}{2} g^{\gamma \sigma}\left(g_{\alpha \sigma, \beta}+g_{\sigma \beta, \alpha}-g_{\alpha \beta, \sigma}\right),
$$

where $g^{\gamma \sigma}$ is inverse to $g_{\alpha \beta}$, that is, $g^{\alpha \sigma} g_{\sigma \beta}=\delta_{\beta}^{\alpha}$. We would like to stress that the affine connections are determined unambiguously irrespective of the dimension of the tangent space.

For constructing gauge invariant Lagrangians we will also need $e_{A}^{\alpha}$ defined as

$$
e_{A}^{\alpha}=g^{\alpha \gamma} e_{A \gamma},
$$

which can be easily seen to satisfy the metricity condition

$$
\partial_{\beta} e_{A}^{\alpha}=-\omega_{\beta A}^{B} e_{B}^{\alpha}-\Gamma_{\beta \nu}^{\alpha} e_{A}^{\nu} .
$$

The soldering form $e_{A}^{\alpha}$ is inverse to $e_{\beta}^{B}$ only if the number of dimensions of the tangent space and manifold match each other. The contraction over the tangent space indices gives

$$
e_{A}^{\alpha} e_{\beta}^{A}=g^{\alpha \gamma} e_{A \gamma} e_{\beta}^{A}=g^{\alpha \gamma} g_{\gamma \beta}=\delta_{\beta}^{\alpha},
$$

however, $e_{A}^{\alpha} e_{\alpha}^{B} \neq \delta_{B}^{A}$. To prove this, let us introduce $N-4$ orthonormal vectors $\mathbf{n}_{\hat{J}}$ orthogonal to the subspace spanned by $\mathbf{e}_{\alpha}$, that is, $\mathbf{n}_{\hat{J}} \cdot \mathbf{e}_{\alpha}=0$ and $\mathbf{n}_{\hat{J}} \cdot \mathbf{n}_{\hat{I}}=\delta_{\hat{J} \hat{I}}$, where $\hat{J}, \hat{I}=5,6, \ldots, N$. The vectors $\mathbf{n}_{\hat{J}}, \mathbf{e}_{\alpha}$ form a complete basis in tangent space and therefore $\mathbf{v}_{A}$ can be expanded as

$$
\mathbf{v}_{A}=v_{A}^{\alpha} \mathbf{e}_{\alpha}+n_{A}^{\hat{J}} \mathbf{n}_{\hat{J}} .
$$


Taking into account (2.8) we have

$$
e_{A \gamma}=\left(\mathbf{v}_{A} \cdot \mathbf{e}_{\gamma}\right)=v_{A}^{\alpha} g_{\alpha \gamma},
$$

and hence, $v_{A}^{\alpha}=g^{\alpha \gamma} e_{A \gamma}=e_{A}^{\alpha}$, that is, the coefficients $v_{A}^{\alpha}$ in (2.17) coincide with soldering form $e_{A}^{\alpha}$. Taking this into account one gets

$$
\eta_{A B}=\mathbf{v}_{A} \cdot \mathbf{v}_{B}=v_{A}^{\alpha} v_{B}^{\beta} g_{\alpha \beta}+n_{A}^{\tilde{J}} n_{\tilde{J} B}=e_{A}^{\alpha} e_{\alpha B}+n_{A}^{\hat{J}} n_{\hat{J} B},
$$

or after raising the tangent space index $B$ we obtain

$$
e_{A}^{\alpha} e_{\alpha}^{B}=\delta_{A}^{B}-n_{A}^{\hat{J}} n_{\hat{J}}^{B} \equiv P_{B}^{A}
$$

where $P_{B}^{A}$ is a projection operator: $P_{C}^{A} P_{B}^{C}=P_{B}^{A}$. The components $n_{A}^{\hat{J}}$ satisfy the following relations

$$
n_{\hat{J}}^{A} e_{A}^{\alpha}=0, \quad n_{\hat{J}}^{A} n_{A}^{\hat{I}}=\delta_{\hat{J}}^{\hat{I}} .
$$

To verify these relations let us consider the expansion

$$
\mathbf{n}_{\hat{J}}=l_{\hat{J}}^{B} \mathbf{v}_{B}
$$

Substituting this expression into $n_{\hat{J} A}=\left(\mathbf{n}_{\hat{J}} \cdot \mathbf{v}_{A}\right)$ we obtain $n_{\hat{J} A}=l_{\hat{J}}^{B} \eta_{B A}$ and hence $l_{\hat{J}}^{B}=n_{\hat{J}}^{B} ;$ therefore

$$
\mathbf{n}_{\hat{J}}=n_{\hat{J}}^{B} \mathbf{v}_{B}=n_{\hat{J}}^{B}\left(e_{B}^{\alpha} \mathbf{e}_{\alpha}+n_{B}^{\hat{I}} \mathbf{n}_{\hat{I}}\right),
$$

from which (2.21) follows immediately.

In vielbiens formalism the soldering form $e_{A}^{\alpha}$ is a fundamental quantity which is required to be invariant under the group of local Lorentz transformations (2.2), where $\Lambda_{A}{ }^{B}=$ $\Lambda_{A}{ }^{B}(x)$. Under Lorentz transformations the basis vectors $\mathbf{v}_{A}$ transform as

$$
\mathbf{v}_{A} \rightarrow \tilde{\mathbf{v}}_{A}=\Lambda_{A}{ }^{B} \mathbf{v}_{B}
$$

and correspondingly

$$
\mathbf{e}_{\alpha}=e_{\alpha}^{B} \mathbf{v}_{B}=e_{\alpha}^{B}\left(\Lambda^{-1}\right)_{B}{ }^{A} \tilde{\mathbf{v}}_{A}=\tilde{e}_{\alpha}^{A} \tilde{\mathbf{v}}_{A} .
$$

It then follows that

$$
e_{\alpha}^{A} \rightarrow \tilde{e}_{\alpha}^{A}=e_{\alpha}^{B}\left(\Lambda^{-1}\right)_{B}{ }^{A}, \quad e_{A}^{\alpha} \rightarrow \tilde{e}_{A}^{\alpha}=\Lambda_{A}{ }^{B} e_{B}^{\alpha} .
$$

The transformation law for the spin-connection follows from its definition:

$$
\tilde{\omega}_{\beta A}{ }^{B} \tilde{\mathbf{v}}_{B}=-\nabla_{\beta} \tilde{\mathbf{v}}_{A}
$$

Substituting $\tilde{\mathbf{v}}_{B}=\Lambda_{B}{ }^{C} \mathbf{v}_{C}$ and taking into account (2.6) we deduce that

$$
\omega_{\beta A}{ }^{B} \rightarrow \tilde{\omega}_{\beta A}{ }^{B}=\left(\Lambda \omega_{\beta} \Lambda^{-1}\right)_{A}{ }^{B}+\left(\Lambda \partial_{\beta} \Lambda^{-1}\right)_{A}{ }^{B},
$$

where $\Lambda$ and $\Lambda^{-1}$ are the matrices corresponding to Lorentz transformation and its inverse. 


\section{Curvature}

To introduce the curvature for the spin-connection, consider the spinors $\psi$ which transform in tangent space according to

$$
\psi \rightarrow \exp \left(\frac{1}{4} \lambda^{A B} \Gamma_{A B}\right) \psi
$$

where $\Gamma_{A B}=\frac{1}{2}\left(\Gamma_{A} \Gamma_{B}-\Gamma_{B} \Gamma_{A}\right)$ are generators of the Lie algebra in the spinor representation and $\Gamma_{A}$ are $N$ Dirac matrices satisfying

$$
\left\{\Gamma^{A}, \Gamma^{B}\right\}=2 \eta^{A B}, \quad \Gamma^{\dagger A}=\Gamma^{0} \Gamma^{A} \Gamma^{0}
$$

The Dirac action

$$
\int d^{4} x \sqrt{g} \bar{\psi} i \Gamma^{C} e_{C}^{\alpha} D_{\alpha} \psi
$$

where

$$
D_{\alpha} \equiv \partial_{\alpha}+\frac{1}{4} \omega_{\alpha}^{A B} \Gamma_{A B}
$$

is invariant under transformations (2.26), (2.28) and (3.1). Notice that hermiticity of the Dirac action in (3.3) is guaranteed by the metricity condition (2.10).

Next construct the spin-connection curvature by considering the commutator of Dirac operators

$$
\left[D_{\alpha}, D_{\beta}\right]=\frac{1}{4} R_{\alpha \beta}{ }^{A B} \Gamma_{A B}
$$

where

$$
R_{\alpha \beta}{ }^{A B}(\omega)=\partial_{\alpha} \omega_{\beta}{ }^{A B}-\partial_{\beta} \omega_{\alpha}^{A B}+\omega_{\alpha}{ }^{A C} \omega_{\beta C}{ }^{B}-\omega_{\beta}{ }^{A C} \omega_{\alpha C}{ }^{B} .
$$

Under Lorentz transformations this spin curvature transforms as

$$
\left(R_{\mu \nu}\right)_{A}{ }^{B} \rightarrow\left(\Lambda R \Lambda^{-1}\right)_{A}{ }^{B}
$$

To relate the spin-connection curvature to the affine connection curvature consider the identity

$$
\partial_{\beta} \partial_{\alpha} e_{A \gamma}-\partial_{\alpha} \partial_{\beta} e_{A \gamma}=0
$$

Substituting here the expression for $\partial e$ from (2.10) and using this metricity condition one more time to express $\partial e$ which appear after taking the derivative, we immediately arrive at the following relation

$$
R_{\alpha \beta}^{A B}(\omega) e_{B \gamma}=R_{\gamma \alpha \beta}^{\rho}(\Gamma) e_{\rho}^{A}
$$

where

$$
R_{\gamma \alpha \beta}^{\rho}(\Gamma)=\partial_{\alpha} \Gamma_{\beta \gamma}^{\rho}-\partial_{\beta} \Gamma_{\alpha \gamma}^{\rho}+\Gamma_{\alpha \kappa}^{\rho} \Gamma_{\beta \gamma}^{\kappa}-\Gamma_{\beta \kappa}^{\rho} \Gamma_{\alpha \gamma}^{\kappa},
$$

is the Riemann curvature. Taking (2.16) into account, we can express the $4 \mathrm{~d}$ Riemann curvature from (3.9) in terms of $R_{\alpha \beta}{ }^{A B}(\omega)$ as

$$
R_{\gamma \alpha \beta}^{\sigma}(\Gamma)=e_{A}^{\sigma} R_{\alpha \beta}^{A B}(\omega) e_{B \gamma}
$$


irrespective of the number of dimensions of the tangent space. Inversely we can express $R_{\alpha \beta}{ }^{A B}(\omega)$ in terms of $R_{\gamma \alpha \beta}^{\sigma}(\Gamma)$ by using (2.20) to obtain

$$
R_{\alpha \beta}^{A B}(\omega)=R_{\alpha \beta}^{A C}(\omega) n_{C}^{\hat{I}} n_{\hat{I}}^{B}+R_{\gamma \alpha \beta}^{\rho}(\Gamma) e_{\rho}^{A} e^{B \gamma} .
$$

Next we will show that the first term on the right hand side of this equation can be entirely expressed in terms of the spin-connections defining the parallel transport of vectors $\mathbf{n}_{\hat{J}}$ in the subspace of tangent space orthogonal to those part spanned by the four coordinate basis vectors $\mathbf{e}_{\alpha}$. These connections, which we denote by $A_{\beta \hat{J}} \hat{I}$ for the reasons which will become clear later, are defined as

$$
\nabla_{\alpha} \mathbf{n}_{\hat{J}}=-A_{\alpha \hat{J}}^{\hat{I}} \mathbf{n}_{\hat{I}}+B_{\alpha \hat{J}}^{\beta} \mathbf{e}_{\beta}
$$

where indices $\hat{J}$ and $\hat{I}$ run over values $5,6, \ldots, N$. These indices are also raised and lowered with the Minkowski metric $\eta_{\hat{I} \hat{J}}$. We now show that $B_{\alpha \hat{J}}^{\beta}=0$ and derive the metricity conditions for $A_{\alpha \hat{J}} \hat{I}$. On one hand

$$
\nabla_{\alpha} \mathbf{v}_{A}=-\omega_{\alpha A}{ }^{B} \mathbf{v}_{B}=-\omega_{\alpha A}{ }^{B}\left(e_{B}^{\gamma} \mathbf{e}_{\gamma}+n_{B}^{\hat{I}} \mathbf{n}_{\hat{I}}\right),
$$

where we have used (2.17) in the last equality, while on the other hand

$$
\begin{aligned}
\nabla_{\alpha} \mathbf{v}_{A} & =\nabla_{\alpha}\left(e_{A}^{\gamma} \mathbf{e}_{\gamma}+n_{A}^{\hat{I}} \mathbf{n}_{\hat{I}}\right)=\left(\partial_{\alpha} e_{A}^{\gamma}+e_{A}^{\beta} \Gamma_{\alpha \beta}^{\gamma}\right) \mathbf{e}_{\gamma} \\
& +\left(\partial_{\alpha} n_{A}^{\hat{I}}-n_{A}^{\hat{J}} A_{\alpha \hat{J}} \hat{I}\right) \mathbf{n}_{\hat{I}}+n_{A}^{\hat{I}} B_{\alpha \hat{I}}{ }^{\beta} \mathbf{e}_{\beta}
\end{aligned}
$$

Using (3.14), (3.15) and (2.15) we deduce that

$$
B_{\alpha \hat{I}}^{\beta}=0 .
$$

Thus, the affine connection of the vector $\mathbf{n}_{\hat{J}}$ lies entirely in the subspace spanned by the basis vectors $\mathbf{n}_{\hat{J}}$. Moreover, as it follows from (3.14) and (3.15) that

$$
\partial_{\alpha} n_{A}^{\hat{I}}=n_{A}^{\hat{J}} A_{\alpha \hat{J}}^{\hat{I}}-\omega_{\alpha A}{ }^{B} n_{B}^{\hat{I}} .
$$

Next let us define

$$
D_{\alpha}(\omega) n_{A}^{\hat{I}} \equiv \partial_{\alpha} n_{A}^{\hat{I}}+\omega_{\alpha A}{ }^{C} n_{C}^{\hat{I}},
$$

and consider the commutator

$$
\left[D_{\alpha}(\omega), D_{\beta}(\omega)\right] n_{A}^{\hat{I}}=R_{\alpha \beta A}{ }^{C}(\omega) n_{C}^{\hat{I}}
$$

On the other hand according to (3.17)

$$
D_{\alpha}(\omega) n_{A}^{\hat{I}}=n_{A}^{\hat{J}} A_{\alpha \hat{J}}^{\hat{I}}
$$

and therefore

$$
\left[D_{\alpha}(\omega), D_{\beta}(\omega)\right] n_{A}^{\hat{I}}=D_{\alpha}(\omega)\left(n_{A}^{\hat{J}} A_{\beta \hat{J}}^{\hat{I}}\right)-(\alpha \leftrightarrow \beta)=n_{A}^{\hat{J}} F_{\alpha \beta \hat{J}}{ }^{\hat{I}}(A),
$$


where

$$
F_{\alpha \beta}^{\hat{I} \hat{J}}(A)=\partial_{\alpha} A_{\beta}^{\hat{I} \hat{J}}-\partial_{\beta} A_{\alpha}^{\hat{I} \hat{J}}+A_{\alpha}^{\hat{I} \hat{L}} A_{\beta \hat{L}}^{\hat{J}}-A_{\beta}^{\hat{I} \hat{L}} A_{\alpha \hat{L}}^{\hat{J}} .
$$

Thus comparing (3.21) and (3.19) we conclude that

$$
R_{\alpha \beta A}{ }^{C}(\omega) n_{C}^{\hat{I}}=n_{A}^{\hat{J}} F_{\alpha \beta \hat{J}}{ }^{\hat{I}}(A)
$$

and using this result in (3.12) we finally obtain

$$
R_{\alpha \beta}^{A B}(\omega)=F_{\alpha \beta}{ }^{\hat{J} \hat{I}}(A) n_{\hat{J}}^{A} n_{\hat{I}}^{B}+R_{\gamma \alpha \beta}^{\rho}(\Gamma) e_{\rho}^{A} e^{B \gamma} .
$$

To get the Lagrangian for the theory we have to build curvature invariants out of $R_{\alpha \beta}{ }^{A B}(\omega)$ and $e_{A}^{\gamma}$. Contracting the tangent space index in $R_{\alpha \beta}{ }^{A B}$ with $e_{A}^{\sigma}$ always removes the $F$ term in (3.24) thanks to (2.21). There exist only one scalar invariant in the linear order in curvature

$$
R_{\alpha \beta}{ }^{A B}(\omega) e_{A}^{\alpha} e_{B}^{\beta}=R(\Gamma),
$$

where $R(\Gamma)$ is the usual scalar curvature of $4 \mathrm{~d}$ manifold which gives us the Einstein action. Second order invariants in curvature which are obtained by contracting $R_{\alpha \beta}{ }^{A B} R_{\gamma \delta}{ }^{C D}$ with four soldering forms $e_{A} e_{B} e_{C} e_{D}$ in all possible combinations of indices $\alpha \beta \gamma \delta$ give us the space-time curvature invariants

$$
R^{2}(\Gamma), R_{\alpha \beta}(\Gamma) R^{\alpha \beta}(\Gamma), R_{\alpha \beta \gamma \delta}(\Gamma) R^{\alpha \beta \gamma \delta}(\Gamma),
$$

and only the contraction of tangent space indices with themselves generate kinetic terms for $A_{\beta}^{\hat{I} \hat{J}}$ :

$$
g^{\alpha \gamma} g^{\beta \delta} R_{\alpha \beta}^{A B}(\omega) R_{\gamma \delta A B}(\omega)=g^{\alpha \gamma} g^{\beta \delta}\left(F_{\alpha \beta}^{\hat{I} \hat{J}}(A) F_{\gamma \delta \hat{I} \hat{J}}(A)\right)+R_{\alpha \beta \gamma \delta}(\Gamma) R^{\alpha \beta \gamma \delta}(\Gamma) .
$$

In this last expression the Yang-Mills kinetic term appears as part of the gravitational curvature square term.

To summarize, the most general action, up to quadratic order in curvature is given by

$$
\begin{gathered}
I=\int d^{4} x \sqrt{-g}\left[\frac{1}{16 \pi G} R_{\alpha \beta}{ }^{A B}(\omega) e_{A}^{\alpha} e_{B}^{\beta}\right. \\
\quad+R_{\alpha \beta}{ }^{A B} R_{\gamma \delta}{ }^{C D}\left(a e_{A}^{\alpha} e_{B}^{\beta} e_{C}^{\gamma} e_{D}^{\delta}+b e_{A}^{\alpha} e_{C}^{\beta} e_{B}^{\gamma} e_{D}^{\delta}+c e_{C}^{\alpha} e_{D}^{\beta} e_{A}^{\gamma} e_{B}^{\delta}\right) \\
\left.\quad-\frac{1}{4} g^{\alpha \gamma} g^{\beta \delta} R_{\alpha \beta}{ }^{A B}(\omega) R_{\gamma \delta A B}(\omega)\right] \\
=\int d^{4} x \sqrt{-g}\left[\frac{1}{16 \pi G} R(\Gamma)+a R^{2}(\Gamma)-b R_{\alpha \beta}(\Gamma) R^{\alpha \beta}(\Gamma)\right. \\
\left.+\left(c-\frac{1}{4}\right) R_{\alpha \beta \gamma \delta}(\Gamma) R^{\alpha \beta \gamma \delta}(\Gamma)-\frac{1}{4} g^{\alpha \gamma} g^{\beta \delta} F_{\alpha \beta} \hat{I} \hat{J}(A) F_{\gamma \delta \hat{I} \hat{J}}(A)\right]
\end{gathered}
$$

where $a, b$, and $c$ are dimensionless constants. We note that it is possible to avoid the ghost in the graviton propagator by choosing the Gauss-Bonnet combination of the curvature square terms which corresponds to the choice $a=\frac{b}{4}=c-\frac{1}{4}$. 
The easiest way to understand the above results which showed that the $\mathrm{SO}(1, N-1)$ invariants split into $\mathrm{SO}(1,3)$ and $\mathrm{SO}(N-4)$ invariants, is to work in a special gauge. We first split the constraint (2.15) for $A=a=1, \ldots, 4$ and $A=\hat{I}=5, \ldots N$ :

$$
\begin{aligned}
& 0=\partial_{\mu} e_{a}^{\nu}+\omega_{\mu a}{ }^{b} e_{b}^{\nu}+\omega_{\mu a}{ }^{\hat{I}} e_{\hat{I}}^{\nu}+\Gamma_{\mu \rho}^{\nu} e_{a}^{\rho} \\
& 0=\partial_{\mu} e_{\hat{I}}^{\nu}+\omega_{\mu \hat{I}}{ }^{a} e_{a}^{\nu}+\omega_{\mu \hat{I}}{ }^{\hat{J}} e_{\hat{J}}^{\nu}+\Gamma_{\mu \rho}^{\nu} e_{\hat{J}}^{\rho}
\end{aligned}
$$

The vielbeins $e_{A}^{\mu}$ transform under $\mathrm{SO}(1, N-1)$ transformations according to

$$
e_{A}^{\mu} \rightarrow \tilde{e}_{A}^{\mu}=\Lambda_{A B} e^{\mu B}
$$

In particular,

$$
e_{\hat{I}}^{\mu} \rightarrow \tilde{e}_{\hat{I}}^{\mu}=\Lambda_{\hat{I} a} e^{\mu a}+\Lambda_{\hat{I} \hat{J}} e^{\mu \hat{J}} .
$$

The action, by construction, is invariant under $\mathrm{SO}(1, N-1)$ rotations. Thus, it is possible to use the gauge invariance and the freedom in the choice of gauge parameters $\Lambda_{\hat{I} a}$ to set $e_{\hat{I}}^{\mu}$ to zero

$$
e_{\hat{I}}^{\mu}=0
$$

This leaves the gauge parameters $\Lambda_{a b}$ and $\Lambda_{\hat{I} \hat{J}}$ arbitrary, corresponding to invariance under the subgroup $\mathrm{SO}(1,3) \times \mathrm{SO}(N-4)$. With this gauge choice we see that equation (3.31) implies

$$
\omega_{\mu \hat{I}}^{a}=0
$$

assuming that $e_{a}^{\mu}$ is invertible. The remaining equation (3.30) can now be solved to give the usual expression for $\omega_{\mu a}{ }^{b}$ in terms of $e_{a}^{\mu}$ and its derivative. In this special gauge $\omega_{\mu \hat{I}}^{\hat{J}}=A_{\mu \hat{I}}^{\hat{J}}$ and

$$
R_{\mu \nu}^{a \hat{I}}=0,
$$

while nonvanishig components of the curvature $R_{\mu \nu}^{a b}$ and $R_{\mu \nu}{ }^{\hat{I} \hat{J}}$ are responsible for the gravity and gauge fields respectively.

Thus, the gauge groups can be considered as subgroup of the Lorentz group of a higher dimensional tangent space. The connections $A_{\alpha}^{\hat{I} \hat{J}}$ transform under $\mathrm{SO}(N-4)$ rotations in a subspace orthogonal to the space spanned by coordinate tangent vectors. The gauge fields come unified with gravity within $\mathrm{SO}(1, N-1)$ Lorentz group. In case $N=5$, the connection $A_{\alpha}^{55}$ vanishes and there are no extra gauge fields in addition to gravity in agreement with [2]. For $N=6$ the connection $A_{\alpha}^{56}$ is a Maxwell field and the local gauge group $\mathrm{SO}(2)$ is obviously isomorphic to the $U(1)$ group of electrodynamics. Thus, electromagnetism is unified with gravity in $\mathrm{SO}(1,5)$ tangent space group. The realistic group which can allow us to unify all known interactions is $\mathrm{SO}(1,13)$. In this case in addition to gravity, the theory describes 45 dynamical gauge fields $A_{\alpha}^{\hat{I} \hat{J}}$ which transform under SO (10) group. 


\section{Fermions}

The matter content of the theory, described by fermions, must be in the fundamental spinor representation of the corresponding Lorentz group $\mathrm{SO}(1, N-1)$. At this point, it is useful to make a scan of possible unification groups by considering various dimensions of the tangent space in four dimensional manifold.

When the tangent space have only one extra dimension compared to the dimension of space-time the tangent group is the de Sitter group $\mathrm{SO}(1,4)$. In this case $\omega_{\mu}^{\hat{5} \hat{5}}=0$ because $\omega_{\mu}^{A B}$ is skew-symmetric in tangent indices and there is no gauge group in addition to the gravity. The spinors are defined in the $\mathrm{SO}(1,4)$ tangent space, where the Majorana condition can be imposed. This case is completely identical to General Relativity with $\mathrm{SO}(1,3)$ tangent group.

For $N=6$ the gauge group is $\mathrm{SO}(2)$ and it describes the Maxwell field. The spinors are in the $\mathrm{SO}(1,5)$ tangent space, where a symplectic-Majorana or Weyl condition can be imposed. The Clifford algebra is then $C l(1,5)=\mathbb{H}(4)$ and the spinor is of dimension 8 . It reduces to two independent spinors when the symplectic-Majorana or Weyl condition is imposed, which are equivalent to a Dirac spinor, or a pair of Majorana spinors with respect to $\mathrm{SO}(1,3)$.

In a seven dimensional tangent space $(N=7)$ the gauge group is $\mathrm{SO}(3)$, which is locally isomorphic to $\mathrm{SU}(2)$. The Clifford algebra of the $\mathrm{SO}(1,6)$ tangent group is $C l(1,6)=\mathbb{C}(8)$ and the spinor is of dimension 8 . No further conditions can be imposed in this case to reduce the number of independent components. The spinor is of the form $\psi_{\alpha i}$ with $i=1,2$ in the spinor representation of $\mathrm{SO}(3)$ and it is a Dirac spinor with respect to the index $\alpha$.

When $N=8$, the gauge group is $\mathrm{SO}(4)$ and the tangent group is $\mathrm{SO}(1,7)$. The Clifford algebra for this tangent group is $C l(1,7)=\mathbb{R}(16)$ and the spinor is of dimension 16. It can be subject to the Weyl condition, thus, reducing the number of independent components to 8. Since $\mathrm{SO}(4)$ is locally isomorphic to $\mathrm{SU}(2) \times \mathrm{SU}(2)$ the spinor is of the form $\psi_{\alpha i}$ and $\psi_{\alpha i^{\prime}}$ where $i=1,2$ and $i^{\prime}=1,2$ are in the spinor representations of the two $\mathrm{SU}(2)$.

Continuing this consideration to higher $N$ we find that the smallest rotation group that has $\mathrm{SU}(3) \times \mathrm{SU}(2) \times \mathrm{U}(1)$ gauge group of the Standard Model as a subgroup is $\mathrm{SO}(10)$ and a good candidate for the realistic model which unifies gravity with gauge interactions is

$$
\mathcal{G}=\mathrm{SO}(1,13)
$$

local symmetry group of the tangent space in the four dimensional manifold. A spinor $\psi_{\widehat{\alpha}}$ in the fundamental representation of $\mathrm{SO}(1,13)$ has $2^{7}=128$ components on which one can impose a Weyl condition

$$
\left(\Gamma_{15}\right)_{\widehat{\alpha}}^{\widehat{\beta}} \psi_{\widehat{\beta}}=\psi_{\widehat{\alpha}}
$$

where $\Gamma_{15}=\Gamma_{0} \Gamma_{1} \cdots \Gamma_{13}$ satisfies $\left(\Gamma_{15}\right)^{2}=1$ and $\Gamma_{0}, \Gamma_{1}, \ldots, \Gamma_{13}$ are fourteen $2^{7} \times 2^{7}$ gamma matrices that satisfy the Clifford algebra $C l(1,13)$. The Weyl condition reduces the number of independent components of the spinor to $\frac{1}{2}(128)=64$. This corresponds to a Dirac $\mathrm{SO}(1,3)$ spinor in the $16_{s}+\overline{16}_{s}$ representation of $\mathrm{SO}(10)$. The 64 independent component spinor describes 32 two components Weyl fermions. Thus, the number of fermions in 
the fundamental spinor representation of $\mathrm{SO}(1,13)$ is twice more than in the Standard Model, where one family contains only 16 Weyl fermions. Majorana condition cannot be imposed in this case and the only way to avoid direct contradiction with experiments is to make half of the fermion very massive using Brout-Englert-Higgs mechanism. This can be easily done by breaking the $\mathrm{SO}(1,13)$ symmetry of tangent group spontaneously to $\mathrm{SO}(1,3) \times \mathrm{SO}(4) \times \mathrm{SO}(6)$ via "scalar" fields which are in the appropriate representations of $\mathrm{SO}(1,13)$. These fields must be coupled to the spinors. One can write few possible such couplings contracting antisymmetric tensors with elements of the Clifford algebra, as for example ([3]),

$$
\psi_{\widehat{\alpha}}\left(C^{\widehat{\alpha} \widehat{\beta}} \phi-\left(C \Gamma^{A B C D}\right)^{\widehat{\alpha} \widehat{\beta}} H_{A B C D}\right) \psi_{\widehat{\beta}}
$$

where $C^{\widehat{\alpha} \widehat{\beta}}$ is the charge conjugation matrix for $\mathrm{SO}(1,13), \phi$ is a scalar field and $H_{A B C D}$ is totally antisymmetric. Let us add to the total action kinetic terms for $\phi$ and for $H_{A B C D}$

$$
g^{\mu \nu} \partial_{\mu} \phi \partial_{\nu} \phi, \quad F_{A B C D E} F^{A B C D E},
$$

where $F_{A B C D E}=D_{[A} H_{B C D E]}$, and a potential term which has a minimum at

$$
\begin{aligned}
\langle\phi\rangle & =v \\
\left\langle H_{a b c d}\right\rangle & =\frac{v}{4 !} \epsilon_{a b c d}, \\
\left\langle H_{\tilde{\imath} \tilde{p} \tilde{m} \tilde{n}}\right\rangle & =\frac{v^{\prime}}{4 !} \epsilon_{\imath \tilde{p} \tilde{m} \tilde{n}},
\end{aligned}
$$

and $\left\langle H_{A B C D}\right\rangle$ is zero otherwise; here the indices $a, b, c, d$ take the values $0,1,2,3$ of $\mathrm{SO}(1,3)$ group and $\tilde{\imath}, \tilde{p}, \tilde{m}, \tilde{n}$ are $\mathrm{SO}(4)$ indices. As a result the symmetry $\mathrm{SO}(1,13)$ is broken to $\mathrm{SO}(1,3) \times \mathrm{SO}(4) \times \mathrm{SO}(10)$. Note that $\mathrm{SO}(4)$ is isomorphic to $\mathrm{SU}(2)_{\mathrm{R}} \times \mathrm{SU}(2)_{\mathrm{L}}$. This insures that the projection $\left(1-\gamma_{5}\right) \psi_{\widehat{\alpha}}$ becomes massive, with a Majorana mass which one can take to be of order of the unification scale, for instance, near the Planck scale. The other combination $\left(1+\gamma_{5}\right) \psi_{\widehat{\alpha}}$ which is a $16_{s}$ spinor of $\mathrm{SO}(10)$ remains massless. After that, we can use a Higgs representation of the form $\Gamma^{A B} H_{A B}$ with the vacuum expectation value (vev)

$$
\left\langle H_{I J}\right\rangle=v^{\prime \prime}\left(\delta_{I}^{5} \delta_{J}^{6}+\delta_{I}^{7} \delta_{J}^{10}+\delta_{I}^{8} \delta_{J}^{9}\right)-I \leftrightarrow J
$$

to break the Pati-Salam group SU (4) to $\mathrm{SU}(3)_{\mathrm{c}} \times U(1)_{\mathrm{c}}$. A further breaking occurs via the field $\Gamma^{A B C D E} H_{A B C D E}$, which acquires vev

$$
\left\langle H_{I J K L M}\right\rangle=\mu\left(\delta_{[I}^{2} \delta_{J}^{4} \delta_{K} \delta_{L}^{9} \delta_{M]}^{10}+i(2 \rightarrow 3)+i(5 \rightarrow 6)-i(9 \rightarrow 8)\right)
$$

and breaks $\mathrm{SU}(2)_{\mathrm{R}} \times U(1)_{\mathrm{c}}$ to $U(1)_{\mathrm{Y}}$. The surviving symmetry is that of the Standard Model $\mathrm{SU}(3)_{\mathrm{c}} \times \mathrm{SU}(2)_{\mathrm{L}} \times U(1)_{\mathrm{Y}}$. The symmetry breaking at the last stage to obtain $\mathrm{SU}(3)_{\mathrm{c}} \times U(1)_{\gamma}$ is done by using the fields

$$
\Gamma^{A} H_{A}, \quad \Gamma^{A B C} H_{A B C}, \quad \Gamma^{A B C D E} H_{A B C D E},
$$

which as a result produces the low energy physics. 


\section{Conclusions}

We have shown that one can unify gauge interactions with gravity by considering higher dimensional tangent spaces in a four dimensional space-time. The gauged tangent space Lorentz group describes simultaneously the symmetry groups of gravity and gauge interactions, provided a metricity condition is satisfied. The spin-connections of the higher dimensional tangent space fully incorporate information on the affine connection of spacetime as well as the gauge fields. Those connections which are responsible for gravity are "composite" because they satisfy extra constraints which allow to express them in terms of the derivatives of the vielbeins. On the other hand the spin-connections responsible for gauge interactions do not obey any constraints and hence are independent. The complete geometric unification of gravity and gauge interactions is realized by writing the action of the theory just in terms of curvature invariants of the tangent group which contains the Yang-Mills action for gauge fields.

The realistic group which unifies the gravity with gauge interactions and contains the Standard Model is $\mathrm{SO}(1,13)$ in a fourteen dimensional tangent space. It corresponds to SO (10) grand unified theory concerning the gauge fields content, however, it has double the number of fermions, half of which can be made very massive via Brout-Englert-Higgs mechanism. The $\mathrm{SO}(1,13)$ is then broken first to $\mathrm{SO}(1,3) \times U(1) \times \mathrm{SU}(2) \times \mathrm{SU}(3)$ and then to $\mathrm{SO}(1,3) \times U(1)_{\mathrm{em}} \times \mathrm{SU}(3)$ by using Brout-Englert-Higgs mechanism. Since the Dirac operator plays a fundamental role in this setting, it is natural to look for connections between this construction and that of noncommutative geometry. In addition, the need to add Higgs scalar fields suggests that a total unification of gravity, gauge and Higgs fields within one geometrical setting, should be possible by replacing the continuous fourdimensional manifold by a noncommutative space which has both discrete and continuous structures [5]. This possibility and others will be the subject of future investigations.

\section{Notes added:}

- After this paper was submitted we were informed by R. Percacci of his work in references [6-8]. In reference [6] a $\mathrm{GL}(4, R)$ model is considered with torsion and a connection with non-metricity. In reference [7] this is generalized to GL $(N, R)$ broken spontaneously to $O(1, N-1)$. In reference [8] the issue of chiral fermions in a gauged $\mathrm{SO}(3,11)$ model broken to $\mathrm{SO}(3,1) \times \mathrm{SO}(10)$ where the Majorana-Weyl condition is imposed to avoid mirror fermions. This model does suffer from the presence of ghosts for scalar Higgs fields and whenever the Minkowski metric is used an odd number of times. Although the methods in these works are similar to the ones presented here, there is little overlap.

- Michel Dubois-Violette, communicated to us the following. In 1970, R. Greene has proved that a 4-dimensional Lorentzian manifold admits locally an isometric smooth free embedding in Minkowski space $M(1,13)$ [9]. There is a similar result proved the same year for the Euclidean signature in M.L. Gromov and V.A. Rokhlin [10]. This means that one can include an arbitrary deformation of the four-manifold in the same flat space and eventually expect to quantize space-time in the fixed Minkowski space $M(1,13)$. 


\section{Acknowledgments}

A.H.C would like to thank the Pauli Center at the Institute for Theoretical Physics, ETH, Zurich, Switzerland and the Arnold Sommerfeld Institute for Theoretical Physics at the Ludwig Maximilians University, Munich, Germany for their hospitality where this research was done. His work is also supported in part by the National Science Foundation Grant No. Phys-1202671 and Phys-1518371. The work of V.M is supported by TRR 33 "The Dark Universe" and the Cluster of Excellence EXC 153 "Origin and Structure of the Universe".

Open Access. This article is distributed under the terms of the Creative Commons Attribution License (CC-BY 4.0), which permits any use, distribution and reproduction in any medium, provided the original author(s) and source are credited.

\section{References}

[1] S. Weinberg, Generalized theories of gravity and supergravity in higher dimensions, in the proceeding of $5^{\text {th }}$ Workshop on Grand Unification, K. Kang et al. eds., World Scientific, Singapore (1984).

[2] A.H. Chamseddine and V. Mukhanov, Gravity with de Sitter and unitary tangent groups, JHEP 03 (2010) 033 [arXiv: 1002.0541] [InSPIRE].

[3] A.H. Chamseddine and J. Fröhlich, $\mathrm{SO}(10)$ unification in noncommutative geometry, Phys. Rev. D 50 (1994) 2893 [hep-th/9304023] [INSPIRE].

[4] C. Misner, K. Thorne and J. Wheeler, Gravitation, Freeman and Company (1993).

[5] A.H. Chamseddine and A. Connes, Noncommutative geometry as a framework for unification of all fundamental interactions including gravity. Part I, Fortsch. Phys. 58 (2010) 553 [arXiv: 1004.0464] [INSPIRE].

[6] R. Percacci, The Higgs phenomenon in quantum gravity, Nucl. Phys. B 353 (1991) 271 [arXiv: 0712.3545] [INSPIRE].

[7] F. Nesti and R. Percacci, Graviweak unification, J. Phys. A 41 (2008) 075405 [arXiv:0706.3307] [INSPIRE].

[8] F. Nesti and R. Percacci, Chirality in unified theories of gravity, Phys. Rev. D 81 (2010) 025010 [arXiv: 0909.4537] [INSPIRE].

[9] R. Greene, Isometric embeddings of Riemannian and pseudo-Riemannian manifolds, Mem. Amer. Math. Soc. 97 (1970).

[10] M.L. Gromov and V.A. Rokhlin, Embeddings and immersions in Riemannian geometry, Uspekhi Mat. Nauk 25 (1970) 1 [Russ. Math. Surv. 25 (1970) 1]. 